\title{
Application of Business Intelligence to the Power System Process Security
}

\author{
N. Jacome-Grajales, G. Escobedo-Briones, G. Arroyo-Figueroa \\ Instituto de Investigaciones Electricas, Gerencia de Sistemas Informaticos, \\ Cuernavaca, Mexico \\ \{nejacome, gescobedo, garroyo\}@iie.org.mx
}

\begin{abstract}
Nowadays, the companies have a growing need for timely and reliable information for operative and strategic decision-making. Companies have understood the importance of enforcing achievements of the goals defined by their business strategies through business intelligence concepts. This paper describes the design and development of business intelligence tools applied to industrial security. The goal is to have available and timely information to make better decisions, to reduce the number of accidents and incidents. The dashboards show a clear and simple way the main indicators of security process. Also, shows the relationship between indicators to determine which may be the attitude possible that caused the accident. The BI tool can be applied in future to other Electric Power Utility processes.
\end{abstract}

Keywords: Information management, decision support systems, business intelligence, industrial security, power system.

\section{Introduction}

In a competitive world, information management becomes strategic. The management information aids the modern executive in the making strategic decision, providing quality information taken from the organization's large volumes of past and present data in a summarized and timely manner. Comprehensive and timely information and knowledge are crucial in improve business operations [1]. Management information and business intelligence play a central role in producing up-to-date information for operative and strategic decision-making.

The conceptualization, planning, implementation, development and putting into operation of enterprise information system and business intelligence, in a Company are not a trivial matter [2]. It brings about countless challenges to organization, administration, training and integration, as well as the resolution of technological and cultural problems. Some of the main problems can be [3]:

There exists a large amount of data but it is not easily accessible. Generally, an executive must turn to other people to obtain data. The time required for a middle- or high-level company manager to obtain the required information to be able to make a decision is long, sometimes days. 
There are same data with different value, the same data from two or more different sources, in general they do not agree. The same data can follow different paths through each of the departments or areas in the organization, from its origin.

There is a lot of data. But what is important is often unknown. The databases tend to grow indiscriminately and as they do, it becomes difficult to access the relevant information for a certain decision making context.

There are some data not very precise. The data can have an error, such as a measurement error; also, the data can be input more than once throughout its different paths, each input can be a potential source of error.

There are large volume of data that is generated daily in the different technical and administrative processes of production and control related to the generation, transmission, transformation and distribution of electric energy, as well as the associated aspects of administration and finances.

There are variety of platforms, operating systems, programming languages and methods of communication between the operational systems. The age of certain operational systems developed with obsolete techniques.

There are lack of methodologies and ways of working, lack of updated information or the lack of documentation of the operational systems, lack of knowledge about the computer information technology culture based on new paradigms.

In this complex scenario, the application of business intelligence technology can be a big task. Business intelligence requires reliable and timely information. Business Intelligence can be defined as a collection of decision support technologies for gathering, providing access to, and analyzing data for the purpose of helping enterprise users (executives, managers and analysts) make better and faster business decisions [4].

This paper present the application of business intelligence tools for providing comprehensive and timely information of security in an Electric Power Utility (EPU).

\section{Business Intelligence}

Business Intelligence is a collection of decision support technologies for gathering, providing access to, and analyzing data for the purpose of helping enterprise users (executives, managers and analysts) make better and faster business decisions [5, 6]. The term implies having a comprehensive knowledge of all of the factors that affect the business. It is imperative that companies have an in depth knowledge about factors such as the customers, competitors, business partners, economic environment, and internal operations to make effective and good quality business decisions. Business intelligence enables firms to make these kinds of decisions. Enterprise aimed at enabling knowledge executives, managers and analysts to make better and faster decisions.

The typical components of Business Intelligence architecture for an Enterprise are the following:

\section{Data Sources}

Data sources can be operational databases, historical data, external data for example, from market research companies or from the Internet), or information from the already existing data warehouse environment. The data sources can be relational databases or any other data structure that supports the line of business applications. They also can 
reside on many different platforms and can contain structured information or unstructured information. Thus, the problems of integrating, cleansing, and standardizing data in preparation for BI tasks can be rather challenging.

\section{Extractions, transformation and loading (ETL)}

Extract-Transform-Load (ETL) refers to a collection of tools that play a crucial role in helping discover and correct data quality issues and efficiently load large volumes of data into the warehouse.

\section{Data Warehouse and data marts}

The data warehouse is the significant component of business intelligence. It is subject oriented, integrated. The data warehouse supports the physical propagation of data by handling the numerous enterprise records for integration, cleansing, aggregation and query tasks. A data mart is a collection of subject areas organized for decision support based on the needs of a given department. The key difference is that the creation of a data mart is predicated on a specific, predefined need for a certain grouping and configuration of select data [7].

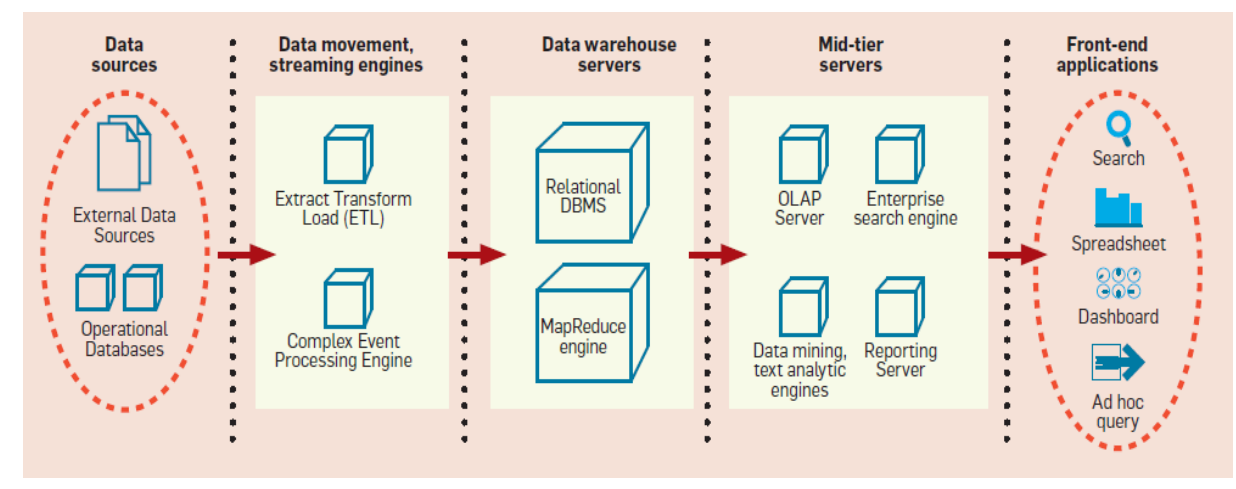

Fig. 1. Typical BI architecture [8].

\section{Data analysis tools}

BI includes several tools for the data analysis. It refers to the way in which business users can slice and dice their way through data using sophisticated tools that allow analytical processing and advance analytics. Online analytic processing (OLAP) provides multidimensional, summarized views of business data and is used for reporting, analysis, modeling and planning for optimizing the business. OLAP tools provide the common BI operations such as filtering, aggregation, drill-down and pivoting. Advanced analytics is referred to as data mining, text analytics, forecasting or predictive analytics and artificial intelligence algorithms, this takes advantage of statistical analysis and artificial intelligence techniques to predict or provide certainty measures on facts.

\section{Front-end-applications}

There are several popular frontend applications through which users perform BI tasks: spreadsheets, enterprise portals for searching, performance management applications 
that enable decision makers to track key performance indicators of the business using visual dashboards, tools that allow users to pose ad hoc queries, viewers for data mining models, and so on. Rapid, ad hoc visualization of data can enable dynamic exploration of patterns, outliers and help uncover relevant facts for BI.

Business intelligence integrates various technologies, such as local and extensive networks, database managers, data visualization, decision support tools, artificial intelligence algorithms and others [9, 10]. Its use depends on the company's specific line business, for this reason each business intelligence application has very particular characteristics for each organization. What makes the concept important is that today the implementation is perfectly feasible given the technological advances in hardware and software that have occurred in the last ten years. These advances include the following among others:

The arrival of On Line Analytical Processing (OLAP) and its application beyond the traditional areas of marketing and finances.

The growth of client/server computing that has created hardware and software servers that are more powerful and sophisticated than ever. Today, the serves compete with the mainframes of yesterday and offer technologically superior memory architectures, with high-speed processor and massive storage capacities.

The appearance of modern database management systems-DBMS's that provide greater support for complex data structures.

The generalized use of internet and intranet accompanied by new and more powerful application.

The advances in data storage, query processing, enterprise search, visualization, data mining, text analytics, artificial intelligence and cloud data services have driven the development of feasible applications.

\section{Industrial Security System}

The management of industrial security has as main objective to preserve the physical integrity, health and welfare of staff. Also, manage health and safety risks at work associated with the processes of the company. An important aspect of security is the prevention and reduction of risks, this is achieved through an existing hazard identified, assess the magnitude of risk and establish controls for inspection, monitoring and implementation of preventive measures for compliance existing regulations. Other action for prevention and reduction of risk is the learning and training for the operation and maintenance of high risk tasks.

To reduce the number and severity of accidents is necessary to have information on the different risks. In order to support safety management, industrial safety system was developed. The industrial safety system, called as Sistema Integral de Seguridad y Salud en el Trabajo (SISST) [11], manages three areas of industrial safety: accident management, infrastructure safety and health protection, safety management. The figure 2 shows the main interface of the system.

\section{Accident module}

The accident module manages of information on accidents, incidents, illnesses, accident cause, management indicators of frequency, severity and degree of risk. 


\section{Infrastructure safety and health protection module}

The accident module manages of information on accidents, incidents, illnesses, accident cause, management indicators of frequency, severity and degree of risk.

\section{Safety management module}

Safety management module manages of information on program evaluations security assessment and compliance with legal requirements, hazard identification, risk assessment and control, and occupational health and preventive-control remedial measures.

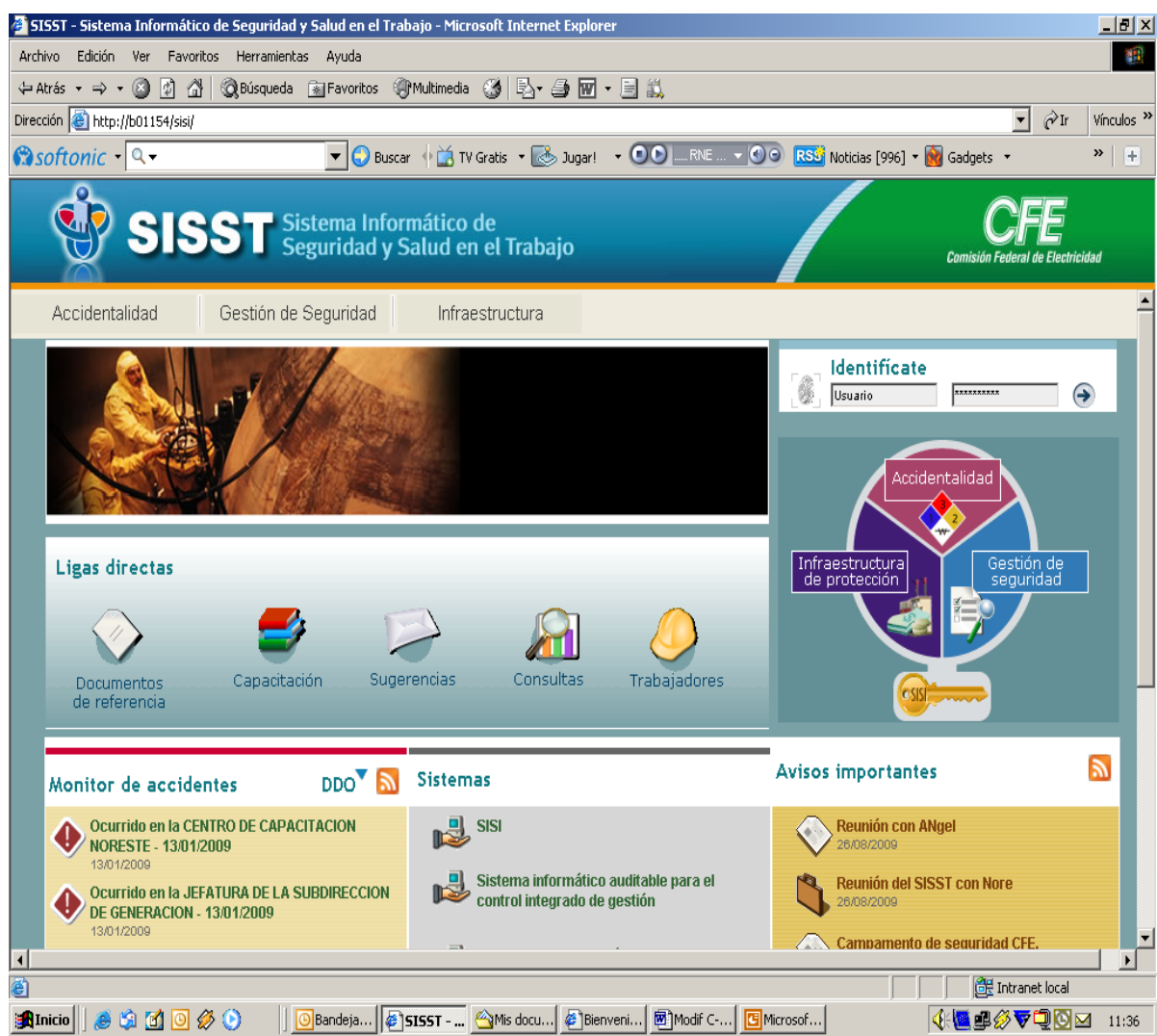

Fig. 2. Security information system.

Although the system handles various standard reports and statistics of accidents to inform at different levels of aggregation, is necessary to relate data for discovery new knowledge about accident and incident. The goal is to design analytical tools that will enable the reduction of accidents. With Business Intelligence tools could devote their efforts to prevent, rather than react. 


\section{Business Intelligence Implementation}

The goal of Business Intelligence is to provide new knowledge to the company, from the automated exploitation of historical information for business actions are taken to be better supported. The results obtained by applying techniques of BI to the database of industrial safety of Mexican Electric Utility are shown in this section.

The benefits it will bring to the implementation of business intelligence technologies are mainly:

a) Accessing security information in a timely and reliable, which allow reducing the time in making and decisions, creating more effective decisions to have the information available.

b) Display detailed information of the security process, making further analysis as a result of having consolidated historical information and current information.

c) Allow delivery of data in a flexible, dynamic and in many cases to solve unplanned queries.

d) As result of above: having a decrease in the number of injured or dead; having a decrease in the economic impact caused by accidents and Decrease the number of days lost due to accidents.

Today there are many tools that offer similar products to both large and small organizations. BI vendors propose solutions both horizontal and vertical and the best choice will depend on the specific need of each organization. With horizontal solutions from scratch by an application tailored to the need. Vertical solutions are aimed at an industry already developed components and only fit specific needs. In this case the implementation of BI tools for the industrial security was done by development proprietary tools under Windows platform and a solution horizontal. For the design and development of BI tools take in count the following queries:

Goal alignment queries: the application of BI tools has the aim to reduce the number of accidents and incidents.

Baseline queries: the source of information is generated by security information system (in Spanish, Sistema de Integral de Seguridad y Salud en el Trabajo -SISST). The system manages three areas of industrial safety for power system processes: accident, infrastructure safety and health protection; and safety management.

Customer and stakeholder queries: There three kinds of users: operative, analytical and executive.

Metric-related queries: the metrics was defined by the security expert. The metrics includes: security indicators, and related variables. A success factor for the development of BI applications is the definition of metrics.

Measurement queries. The operational variables are load by the security information system. There are procedures and methodologies defined by the company for the variables and its load frequency.

The methodology used for the definition of the BI architecture considers the construction of a single enterprise data warehouse and from it will emerge Data Marts (small units of analysis), with an overall vision of the company. The figure 3 shows the BI-SISST architecture. 


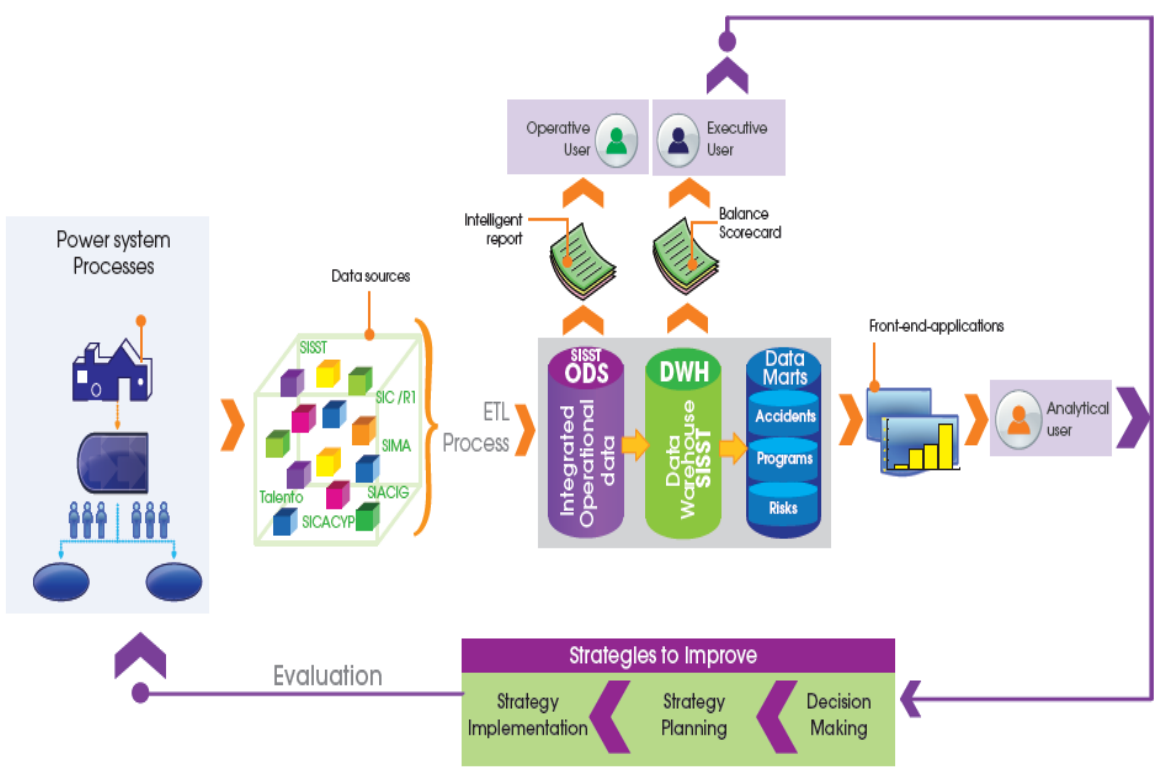

Fig. 3. BI-SISST proposed architecture.

The primary tasks include gathering, preparing and analyzing data. From operational database of industrial security system, extract the most relevant data to the user through an ETL process, which loads and makes the necessary transformations and data cleansing. These are the integrated operational data (ODS-SISST) of the security system. The data itself must be of high quality.

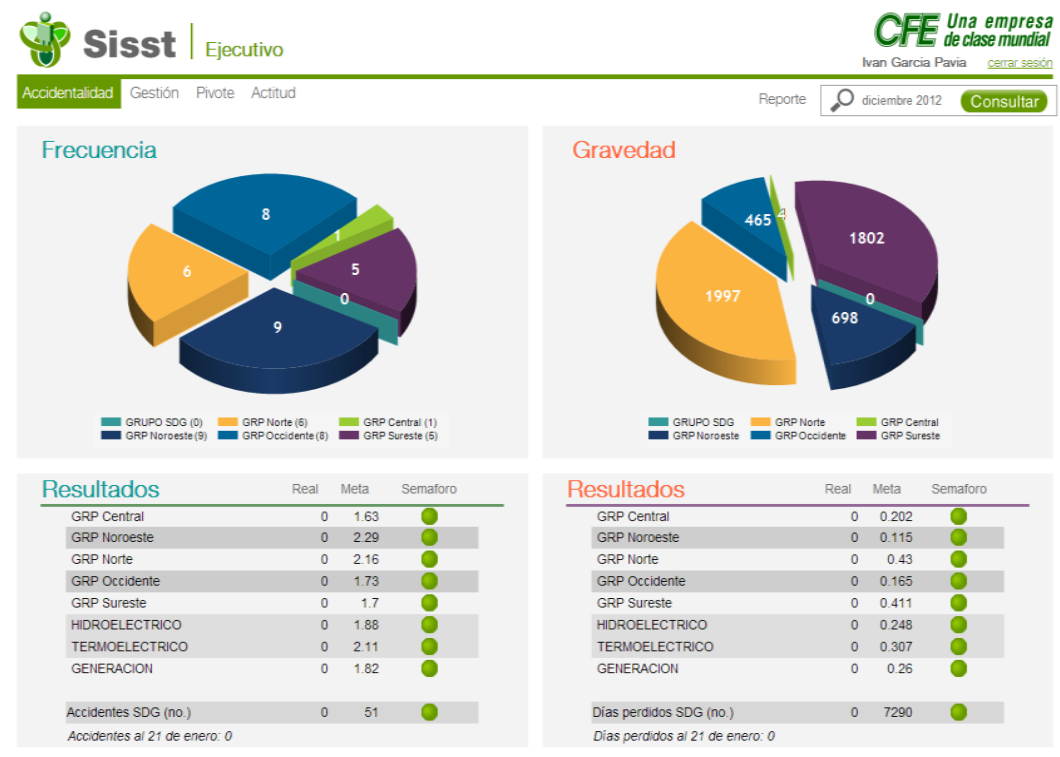

Fig. 4. Accident frequency and severity. 
Once the data is complete and clean, they pass through another ETL process to data warehouse (DWH-SISST). Finally, data marts are created for accidents, safety programs and hazards. From these data were carried out the data analysis, through multidimensional analysis and consulted manager dashboards.

The results of the BI application are the front-end-applications through of dashboards. The Dashboard should provide the executive with a tool for navigating through company's information. The figure 4 shows the accident frequency by area with pie contribution and table of results with goal and indicator state. Also shows the severity by area.

The figure 5 shows the historical, real and forecast accident bye year and by moth. This information is obtained to accident module.

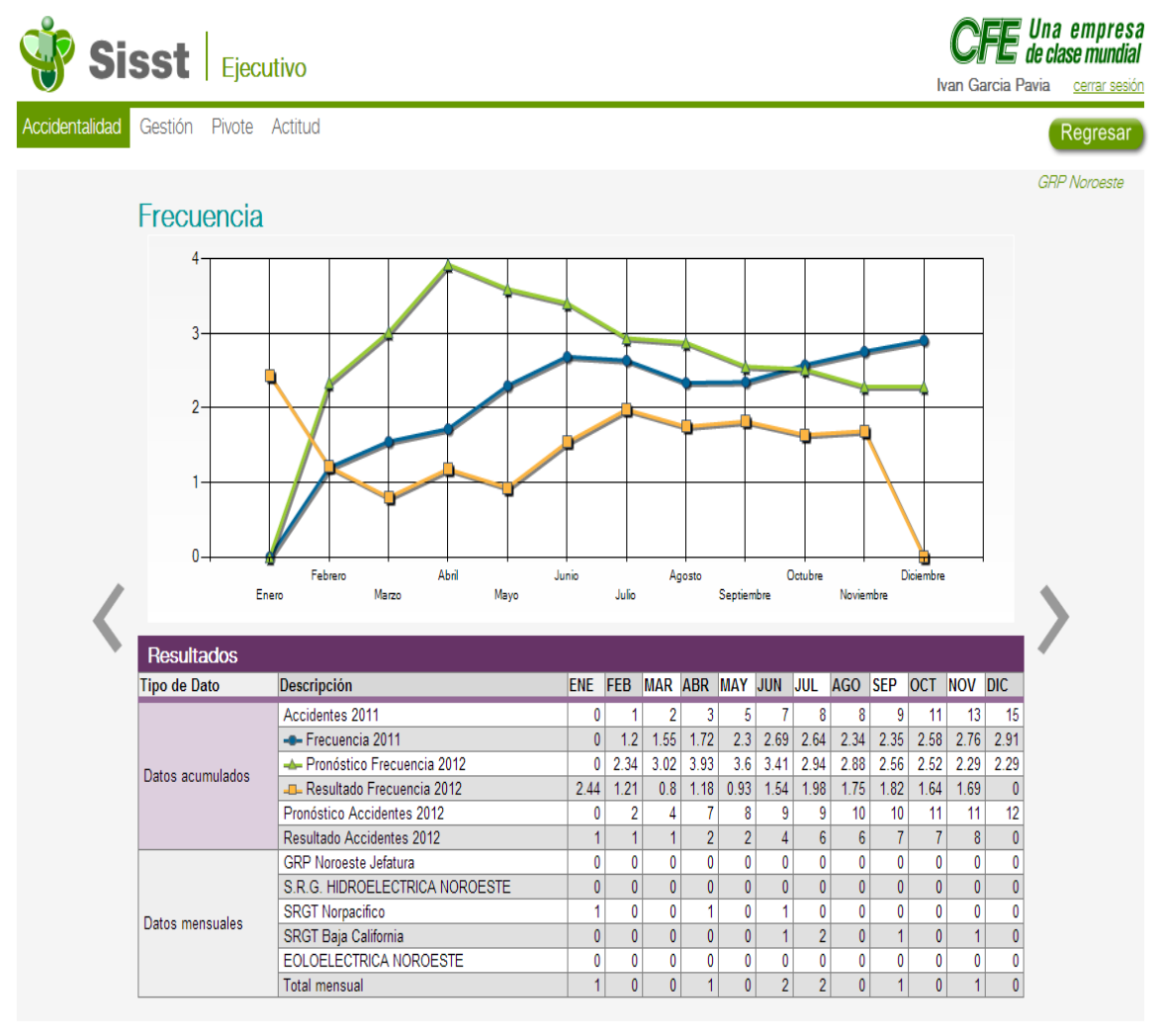

Fig. 5. Accident frequency: historical, real and forecast.

The main indicators of the security have presented in the figure 6 . The definition of indicators is an important task to ensure the success of business intelligence. This dashboard shows the security maturity in terms of identification of hazards, legal requirements, compliance of security programs, safety forms, incidents and acts of government.

With the business intelligence is possible to combine different information. For example it is possible to relate the accident with the attitudes. For this case, the figure 7 presents the relations between the accidents with attitudes. 


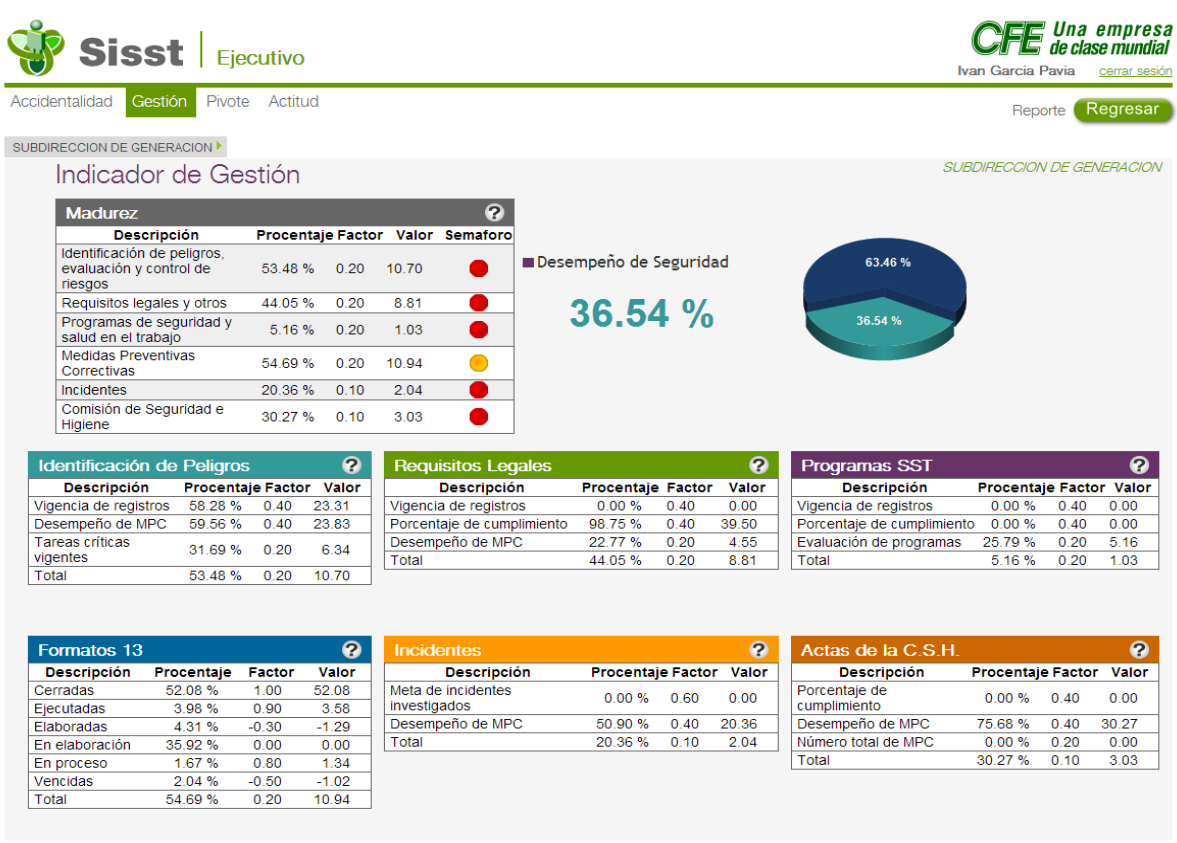

Fig. 6. Management indicators.
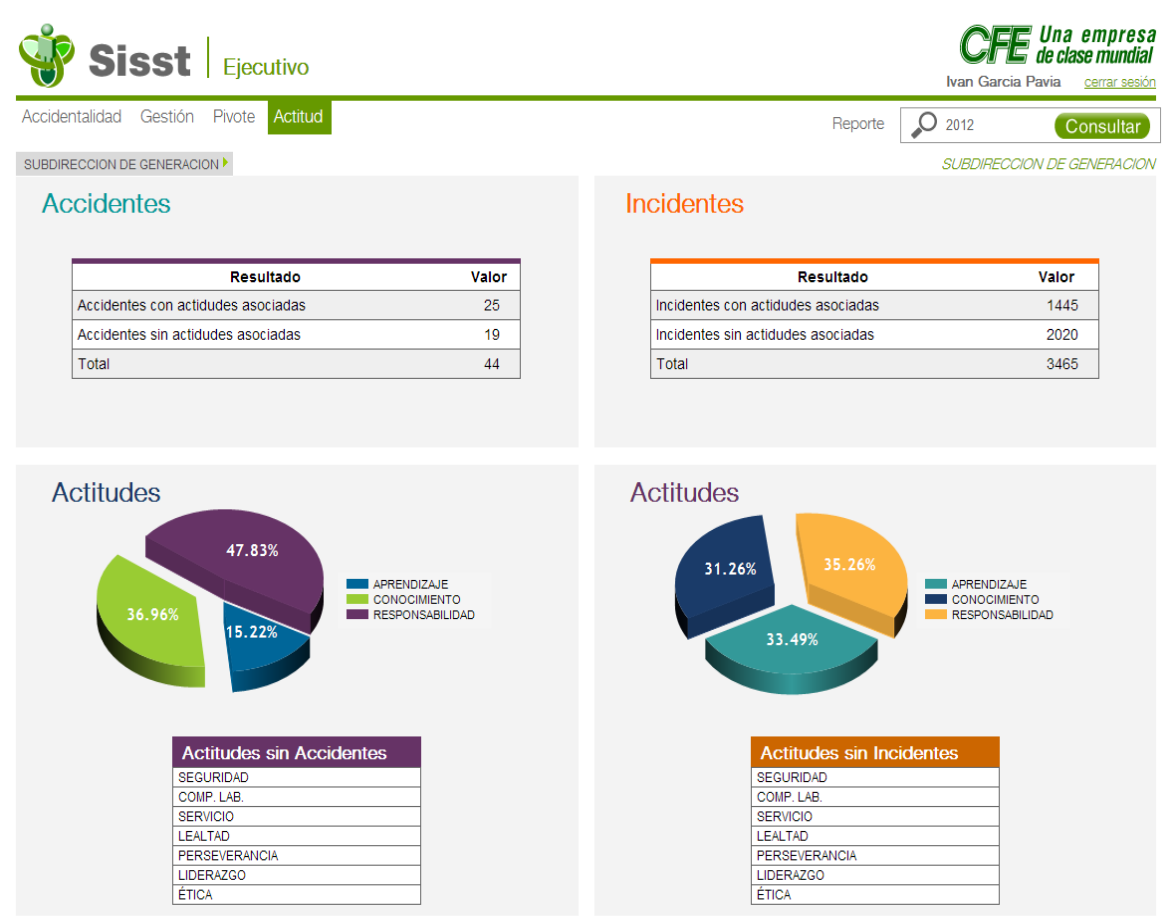

Incidentes

\begin{tabular}{|lc|}
\hline \multicolumn{1}{|c|}{ Resultado } & Valor \\
\hline Incidentes con actidudes asociadas & 1445 \\
\hline Incidentes sin actidudes asociadas & 2020 \\
\hline Total & 3465 \\
\hline
\end{tabular}

Fig. 7. Relationship between accidents and incidents with attitudes. 
Also, it is possible to classify the kind of attitudes: learning, knowledge and responsibility. This means that the an accident occurs can determine if the accident occurred due to lack of training, lack of knowledge or lack of responsibility. For the case of incidents also it is possible determinate if occurred by lack of training, knowledge or responsibility.

The capabilities of business intelligence include decision support, online analytical processing, statistical analysis, forecasting and data mining. BI-SISST system is a traditional business intelligence application with back-end database and front-end user interface, software that processes the information and reporting systems.

\section{Conclusions}

Business Intelligence as a concept is becoming more common in everyday business life. BI incorporates people, process, and also knowledge as an end product. The implementation of business intelligence (BI) system is a complex undertaking requiring considerable resources.

An important factor to build BI applications is the information management. BI requires reliable and timely information and generates summary information for the operative and strategic decision making. In addition, the implementation of a BI system is often associated with the following challenges: underlying original back-end systems and processes which were not adapted for BI applications; poor data quality derived from source systems that can often go unnoticed until cross-systems analysis is conducted; and the maintenance process that tends to be vague and ill-defined

To attack this problem is necessary to implement enterprise architecture with its two main components: business architecture and technological architecture can help ensure that the data source will be reliable.

The business intelligence tools developed for the industrial security have had good results. The information displayed through dashboards make career choices have led to the decrease of accidents. In particular, the relationship between accidents and attitudes has been a great help to generate preventive actions to avoid accidents. Also, it indicates if the accident occurred due to lack of training, knowledge, or lack of responsibility.

Acknowledgment. The authors wish to thank Israel Paredes Rivera, Department Head of Technical Services Unit of CFE for their important work in supporting, organizing and promoting the project. We would like to convey special thanks to all the operators who selflessly shared their reliable knowledge and experience to make this project successful.

\section{References}

1. Chaudhuri, S., Dayal, U., Narasayya, V.: An Overview of Business Intelligence Technology. Communications of the ACM, Vol. 54, No. 8, pp: 88-98 (2011)

2. Hannula, M., Pirttimaki, V.: Business intelligence Emperical Study on to the 50 Finnish Companies. Journal of American Academy of Business, Vol. 2, No. 2 (2003) 
3. Mejia-Lavalle, M., Arroyo-Figueroa, G.: Construction of a Corporative Information System for an Electric Power Company. IEEE Conference on Intelligent Engineering Systems Proceedings, pp. 177-182 (2010)

4. Ranjan, J.: Business Intelligence: concepts, components, techniques and benefits. Journal of Theoretical and Applied Information Technology, pp. 60-69 (2007)

5. Turban, E., Aronson, J., Liang, T., Shard, R.: Decision support and business intelligence systems. Pretince Hall (2005)

6. McKay, L.: Businees Intelligence comes out of the back office. CRM Magazine (2008)

7. Rizzi, S., Abelló, A., Lechtenborger, J., Trujillo, J.: Research in Data Warehouse Modeling and Design: Dead or Alive? In 9th International Workshop on Data Warehousing and OLAP (DOLAP), Arlington (USA), ACM Press, pp. 3-10 (2006)

8. Watson, H.J., Wixom, B.H.: The current state of business intelligence. Computer, Vol. 40, No. 9, pp. 96-99 (2007)

9. Olszak, C., Ziemba, E.: Approach to Building and Implementing Business Intelligence Systems. Interdisciplinary Journal of Information, Knowledge, and Management, Vol. 2, pp: $135-148$ (2007)

10. Wang, H., Wang, S.: A Knowledge Management Approach to Data Mining Process for Business Intelligence. Industrial Management \& Data Systems, Vol. 108, No. 5, pp. 622$634(2008)$

11. Jacome-Grajales, N., Escobedo-Briones, G., Guadarrama Villa, E.: Inteligencia de Negocios en el área de seguridad de la CFE. Congreso Internacional sobre Innovación y Desarrollo Tecnológico, pp. 677-685 (2011) 